\title{
EFFECT OF ROTATION IN AN ORTHOTROPIC ELASTIC SLAB
}

\author{
S. SANTRA* \\ Department of Mathematics \\ Gargi Memorial Institute of Technology \\ Kolkata-700144, INDIA \\ E-mail: sutapasantra.ismu.math@gmail.com
}

\author{
A. LAHIRI \\ Department of Mathematics, Jadavpur University \\ Kolkata-700032, INDIA \\ E-mail: lahiriabhijit2000@yahoo.com \\ N.C. DAS \\ Department of Mathematics \\ Brainware Group of Institutions \\ Barasat- 700127, INDIA \\ E-mail: ncdasmaths@gmail.com
}

\begin{abstract}
The fundamental equations of the two dimensional generalized thermoelasticity (L-S model) with one relaxation time parameter in orthotropic elastic slab has been considered under effect of rotation. The normal mode analysis is used to the basic equations of motion and heat conduction equation. Finally, the resulting equations are written in the form of a vector-matrix differential equation which is then solved by the eigenvalue approach. The field variables in the space time domain are obtained numerically. The results corresponding to the cases of conventional thermoelasticity CTE), extended thermoelasticity (ETE) and temperature rate dependent thermoelasticity (TRDTE) are compared by means of graphs.
\end{abstract}

Key words: Orthotropic elastic slab, normal mode analysis, effect of rotation.

\section{Introduction}

Most of the thermoelasticity and generalized thermoelasticity (coupled or uncoupled) problems have been solved by using potential functions. This method is not always suitable as discussed by Sherief [1] and Sherief and Anwar [2]. It may be summarized by (i) the boundary and initial conditions for physical problems are directly related to the physical quantities under consideration and not to the potential function and (ii) the solution of the physical problem in natural variables is convergent while other potential function representations are not always convergent. The alternative to the potential function approach are - (i) StateSpace approach: This method is essentially an expansion in a series in terms of the coefficient matrix of the field variables in ascending powers and applying the Caley-Hamilton theorem, which requires extensive algebra, and (ii) Eigenvalue approach:

In this method the basic equations are written in the form of a vector matrix differential equation which reduces it to an algebraic eigenvalue problem and the solutions for the field variables are achieved by determining the eigenvalues and the corresponding eigenvectors of the coefficient matrix. In the eigenvalue approach the physical quantities such as material constants are directly involved in the formulation of the

\footnotetext{
* To whom correspondence should be addressed
} 
problem and as such the boundary and initial conditions can be applied directly. In this theory body forces and/or heat sources are also accommodated (Das and Lahiri [3], Bachher et al. [4], Kar and Kanoria [5]).

The classical theory of coupled and uncoupled thermoelasticity predicts two phenomena not compatible with physical observation. The heat conduction equation is of dissuasion type, which does not contain any elastic terms contrary to the fact that the elastic changes produce infinite speed of propagation of thermal waves. The equation of motion is of a hyperbolic type transmitting finite speed of elastic waves in the medium. To overcome these problems, Biot [6] introduced coupled thermoelasticity. In this theory, the governing equations are coupled, eliminating the paradox of the classical theory. To eliminate the second shortcoming, the theory of generalized thermoelasticity was developed. The generalized thermoelasticity theories admit so-called second sound effects, which predict a finite velocity of propagation of the heat flux. At present there are two different theories of generalized thermoelasticity: (i) Lord and Shulman (L-S theory) [7] for the special case of isotropic body, and (ii) Green and Lindsay (G-L theory) [8]. The L-S model itself is based on a modified Fourier's law and the obtained wave-type heat conduction equation. The L-S model contains the heat flux vector and its time derivative. In this case a new constant arises that acts as a time relaxation parameter. This theory was extended by Dhaliwal and Sherief [9] to include the anisotropic case. The uniqueness of solution for this theory was proved under different conditions by Ignaczak [10, 11], by Sherief and Dhaliwal [12] and by Sherief [13]. In the G-L theory there are two relaxation time parameters and they modify not only the heat conduction equation but also all the equations of the coupled theory without violating Fourier's law. Both theories are structurally different and one cannot be obtained as a particular case of the other.

\section{Formulation of the problem}

We consider a plane strain problem in a homogeneous infinite slab with thickness $2 h$. the origin is located at the middle of the slab, the $y$-axis along the length and the $z$-axis along the height of the slab.

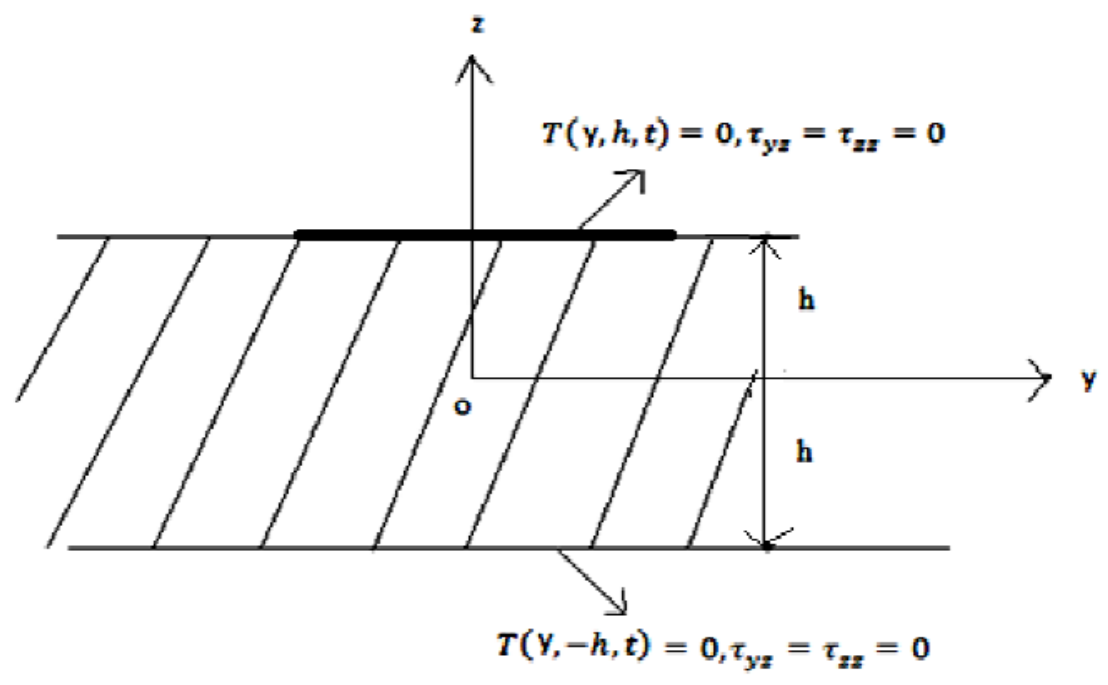

Fig.1. Orthotropic elastic slab.

The equations of motion and heat conduction equation in a non-rotating generalized thermoelastic medium are

$$
\tau_{i j, j}=\rho \ddot{u}_{i},
$$




$$
k_{i j} T_{, i j}-\rho c_{e}\left(\dot{T}+\alpha_{0} \ddot{T}\right)=T_{0} \beta_{i j}\left(\dot{u}_{i, j}+\tau \ddot{u}_{i, j}\right)
$$

where

$$
\begin{aligned}
& \tau_{i j}=A_{i j k l} e_{k l} \delta_{i j}-\beta_{i j}\left(1+\alpha \frac{\partial}{\partial t}\right) T, \\
& \beta_{i j}=A_{i j k l} \alpha_{k l}, \quad e_{i j}=\frac{1}{2}\left(u_{i, j}+u_{j, i}\right), \quad i, j, k, l=1,2,3 .
\end{aligned}
$$

The usual summation convention holds in the analysis. The common notation is usual for special derivatives and a superposed dot for time derivatives.

Equation (2.1) can now be modified in a rotating medium as

$$
\tau_{i j, j}=\rho\left[\ddot{\boldsymbol{u}}_{i}+\{\boldsymbol{\Omega} \times(\boldsymbol{\Omega} \times \boldsymbol{u})\}+(2 \boldsymbol{\Omega} \times \dot{\boldsymbol{u}})\right] .
$$

The displacement vector is measured from a steady state deformed position and the deformation is assumed to be small. The last two terms on the right hand side of Eqs (2.4) appear due to centripetal and Coriolis acceleration, respectively, in a rotating medium.

Since we consider an orthotropic elastic medium in two dimensions subject to the plane-strain parallel to the $y-z$ plane, we assume

$$
u_{1}(y, z, t)=u=0, \quad u_{2}(y, z, t)=v(y, z, t), \quad u_{3}(y, z, t)=w(y, z, t)
$$

where $u, v$ and $w$ are displacements in the $x, y$ and $z$ directions, respectively. Akoz [14]

The corresponding stresses can now be obtained from Eq.(2.3) and are also available in Tauchert and

$$
\begin{aligned}
& \tau_{x x}=A_{12} \frac{\partial v}{\partial y}+A_{13} \frac{\partial w}{\partial z}-\beta_{1}\left(1+\alpha \frac{\partial}{\partial t}\right) T, \\
& \tau_{y y}=A_{22} \frac{\partial v}{\partial y}+A_{23} \frac{\partial w}{\partial z}-\beta_{2}\left(1+\alpha \frac{\partial}{\partial t}\right) T, \\
& \tau_{z z}=A_{23} \frac{\partial v}{\partial y}+A_{33} \frac{\partial w}{\partial z}-\beta_{3}\left(1+\alpha \frac{\partial}{\partial t}\right) T,
\end{aligned}
$$

and

$$
\tau_{y z}=A_{44}\left(\frac{\partial v}{\partial z}+\frac{\partial w}{\partial y}\right)
$$
in the form

Then the corresponding displacement equations of motion in a rotating medium follow from Eq.(2.5) 


$$
\begin{aligned}
& A_{22} \frac{\partial^{2} v}{\partial y^{2}}+A_{44} \frac{\partial^{2} w}{\partial z^{2}}+\left(A_{23}+A_{44}\right) \frac{\partial^{2} w}{\partial y \partial z}=\rho\left[\frac{\partial^{2} v}{\partial t^{2}}-\Omega^{2} v-2 \Omega \dot{w}\right]+\beta_{2} \frac{\partial}{\partial y}\left(1+\alpha \frac{\partial}{\partial t}\right) T \\
& A_{44} \frac{\partial^{2} w}{\partial y^{2}}+A_{33} \frac{\partial^{2} v}{\partial z^{2}}+\left(A_{23}+A_{44}\right) \frac{\partial^{2} v}{\partial y \partial z}=\rho\left[\frac{\partial^{2} w}{\partial t^{2}}-\Omega^{2} w+2 \Omega \dot{v}\right]+\beta_{3} \frac{\partial}{\partial z}\left(1+\alpha \frac{\partial}{\partial t}\right) T
\end{aligned}
$$

where $\boldsymbol{\Omega}=(\Omega, 0,0)$. In a non-rotating medium $\Omega \equiv 0$, Eqs (2.11) and (2.12) reduce to these of Das et al. [15].

The temperature field $T(y, z, t)$ is assumed to satisfy the heat conduction Eq.(2.2) as proposed by Lord and Shulman [7], so that

$$
k_{y} \frac{\partial^{2} T}{\partial y^{2}}+k_{z} \frac{\partial^{2} T}{\partial z^{2}}=\rho c_{e}\left(\frac{\partial}{\partial t}+\alpha_{0} \frac{\partial^{2}}{\partial t^{2}}\right) T+T_{0}\left(\frac{\partial}{\partial t}+\tau \frac{\partial^{2}}{\partial t^{2}}\right)\left(\beta_{2} \frac{\partial v}{\partial y}+\beta_{3} \frac{\partial w}{\partial z}\right)
$$

Equations (2.11)-(2.13) form a coupled system to represent coupled thermal, dilatational and shear waves in a rotating elastic medium. The thermal field effects the shear motion due to rotation.

\section{Formulation of vector-matrix differential equation}

We apply the normal mode analysis defined by

$$
\left[v, w, T, \tau_{i j}\right](y, z, t)=\left[u^{*}, v^{*}, T^{*}, \tau_{i j}^{*}\right] e^{\omega t+i a y} .
$$

Using the above transformation to Eqs (2.11) - (2.13), we get the following equations

$$
\begin{aligned}
& -\left(A_{22} a^{2}+\rho\left(\omega^{2}-\Omega^{2}\right)\right) V^{*}+2 \omega \rho \Omega w^{*}-i a \beta_{2}^{\prime} T^{*}+A_{44} \frac{d^{2} v^{*}}{d z^{2}}+\left(A_{23}+A_{44}\right) i a \frac{d w^{*}}{d z}=0, \\
& -\left(A_{44} a^{2}+\rho\left(\omega^{2}-\Omega^{2}\right)\right) w^{*}-2 \omega \rho \Omega v^{*}-\beta_{3}^{\prime} \frac{d T^{*}}{d z}+A_{33} \frac{d^{2} w^{*}}{d z^{2}}+\left(A_{23}+A_{44}\right) i a \frac{d v^{*}}{d z}=0 \\
& \left(-k_{y} a^{2}-\rho c_{e}^{\prime} \omega\right) T^{*}+k_{z} \frac{d^{2} T^{*}}{d z^{2}}-i a \beta_{2} T_{0}^{\prime} \omega v^{*}-T_{0}^{\prime} \omega \beta_{3} \frac{d w^{*}}{d z}=0
\end{aligned}
$$

where

$$
\beta_{2}^{\prime}=\beta_{2}(1+\alpha \omega), \quad \beta_{3}^{\prime}=\beta_{2}(1+\alpha \omega), \quad c_{e}^{\prime}=c_{e}\left(1+\alpha_{0} \omega\right), \quad T_{0}^{\prime}=T_{0}(1+\tau \omega),
$$

Equations (3.2)-(3.4) can be written in the form of vector matrix differential equations as follows

$$
\frac{d \bar{V}}{d z}=\tilde{A} \bar{V}
$$

where, 


$$
\bar{V}=\left[v, w, T, v^{\prime}, w^{\prime}, T^{\prime}\right]^{T}
$$

The primes denotes differentiation with respect to $z$, and the matrix A is given by

$$
\tilde{A}=\left[\begin{array}{cccccc}
0 & 0 & 0 & 1 & 0 & 0 \\
0 & 0 & 0 & 0 & 1 & 0 \\
0 & 0 & 0 & 0 & 0 & 1 \\
v_{41} & v_{42} & v_{43} & 0 & v_{45} & 0 \\
v_{51} & v_{52} & 0 & v_{54} & 0 & v_{56} \\
v_{61} & 0 & v_{63} & 0 & v_{65} & 0
\end{array}\right]
$$

where

$$
\begin{aligned}
& v_{41}=\frac{A_{22} a^{2}+\rho\left(\omega^{2}-\Omega^{2}\right)}{A_{44}}, \quad v_{42}=-\frac{2 \Omega \rho \omega}{A_{44}}, \quad v_{43}=\frac{i a \beta_{2}^{\prime}}{A_{44}}, \quad v_{45}=-\frac{i a\left(A_{23}+A_{44}\right)}{A_{44}}, \\
& v_{51}=\frac{2 \Omega \rho \omega}{A_{33}}, \quad v_{52}=\frac{A_{44} a^{2}+\rho\left(\omega^{2}-\Omega^{2}\right)}{A_{33}}, \quad v_{54}=-\frac{i a\left(A_{23}+A_{44}\right)}{A_{33}}, \quad v_{56}=\frac{\beta_{3}^{\prime}}{A_{33}}, \\
& v_{61}=\frac{i a \beta_{2} T_{0}^{\prime} \omega}{k_{z}}, \quad v_{63}=\frac{k_{y} a^{2}+\rho c_{e}^{\prime} \omega}{k_{z}}, \quad v_{65}=\frac{T_{0}^{\prime} \omega \beta_{3}}{k_{z}}
\end{aligned}
$$

\section{Solution of the vector matrix differential equation}

The characteristic equation of the matrix $\tilde{A}$ takes the form

$$
\begin{aligned}
& \lambda^{6}-\lambda^{4}\left(v_{41}+v_{52}+v_{63}+v_{45} v_{54}+v_{56} v_{65}\right)-\lambda^{3}\left(v_{42} v_{54}+v_{45} v_{51}\right)+ \\
& +\lambda^{2}\left(v_{52} v_{63}+v_{41} v_{52}+v_{41} v_{63}-v_{43} v_{61}-v_{42} v_{51}+v_{45} v_{54} v_{63}-v_{45} v_{56} v_{61}+\right. \\
& \left.+v_{65} v_{41} v_{56}-v_{65} v_{43} v_{54}\right)+\lambda\left(v_{42} v_{54} v_{63}-v_{42} v_{56} v_{61}+v_{51} v_{45} v_{63}-v_{51} v_{43} v_{65}\right)+ \\
& +\left(v_{63} v_{42} v_{51}-v_{63} v_{41} v_{52}+v_{43} v_{52} v_{61}-v_{41} v_{52} v_{63}\right)=0 .
\end{aligned}
$$

The roots of the characteristic Eq.(4.1) which are also eigenvalues of $A$ are of the form

$$
\lambda= \pm \lambda_{1}, \quad \lambda= \pm \lambda_{2}, \quad \lambda= \pm \lambda_{3} .
$$

The eigenvector $\tilde{X}=\left[X_{1}, X_{2}, X_{3}, X_{4}, X_{5}, X_{6}\right]^{T}$ corresponding to the eigenvalue $\lambda$ can be calculated as 


$$
\tilde{X}=\left[\begin{array}{c}
\lambda^{2}\left(v_{45} v_{56}+v_{43}\right)+\lambda v_{42} v_{56}-v_{43} v_{52} \\
\lambda^{3} v_{56}+\lambda\left(v_{43} v_{54}-v_{41} v_{56}\right)+v_{43} v_{51} \\
\lambda^{4}-\lambda^{2}\left(v_{41}+v_{52}+v_{45} v_{54}\right)-\lambda\left(v_{42} v_{54}+v_{45} v_{51}\right)+v_{41} v_{52}-v_{42} v_{51} \\
\lambda\left(\lambda^{2}\left(v_{45} v_{56}+v_{43}\right)+\lambda v_{42} v_{56}-v_{43} v_{52}\right) \\
\lambda\left(\lambda^{3} v_{56}+\lambda\left(v_{43} v_{54}-v_{41} v_{56}\right)+v_{43} v_{51}\right) \\
\lambda\left(\lambda^{4}-\lambda^{2}\left(v_{41}+v_{52}+v_{45} v_{54}\right)-\lambda\left(v_{42} v_{54}+v_{45} v_{51}\right)+v_{41} v_{52}-v_{42} v_{51}\right)
\end{array}\right] .
$$

From Eq.(4.3) the eigenvector $\tilde{X}$ corresponding to the eigenvalue $\lambda=\lambda_{i}$ can easily be calculated. We see the following notations

$$
\begin{array}{ll}
X_{1}=[X]_{\lambda=\lambda_{1}}, & X_{2}=[X]_{\lambda=\lambda_{2}}, \quad X_{3}=[X]_{\lambda=\lambda_{3}}, \\
X_{4}=[X]_{\lambda=\lambda_{4}}, \quad X_{5}=[X]_{\lambda=\lambda_{5}}, \quad X_{6}=[X]_{\lambda=\lambda_{6}} .
\end{array}
$$

Using the method as in Das et al. [15] the solution of Eq.(3.6) is

$$
\bar{V}=\sum_{i=1}^{6} a_{i} X_{i} e^{z \lambda_{i}}
$$

The constants are to be determined from the boundary conditions. The displacement components, temperature and stresses can now be written as

$$
\begin{aligned}
& v^{*}=\sum_{i=1}^{6} a_{i}\left[\lambda_{i}^{2}\left(v_{45} v_{56}+v_{43}\right)+\lambda_{i} v_{42} v_{56}-v_{43} v_{52}\right] e^{z \lambda_{i}}, \\
& w^{*}=\sum_{i=1}^{6} a_{i}\left[\lambda_{i}^{3} v_{56}+\lambda_{i}\left(v_{43} v_{54}-v_{41} v_{56}\right)+v_{43} v_{51}\right] e^{z \lambda_{i}}, \\
& T^{*}=\sum_{i=1}^{6} a_{i}\left[\begin{array}{c}
\left.\lambda_{i}^{4}-\lambda_{i}^{2}\left(v_{41}+v_{52}+v_{45} v_{54}\right)-\lambda_{i}\left(v_{42} v_{54}+v_{45} v_{51}\right)+\right] e^{z \lambda_{i}}, \\
+\left(v_{41} v_{52}-v_{42} v_{51}\right)
\end{array}\right] \\
& \tau_{z z}^{*}=A_{23} i a v^{*}+A_{33} \frac{d w^{*}}{d z}-\beta_{3}^{\prime} T^{*}, \\
& \tau_{y z}^{*}=A_{44}\left(i a v^{*}+\frac{d w^{*}}{d z}\right) .
\end{aligned}
$$




\section{Boundary conditions}

We assume that the surface of the slabs are stress-free

i.e, $\quad \tau_{z z}=\tau_{y z}=0 \quad$ at $\quad z= \pm h$,

we further assume the temperature distribution over the upper and lower surface of the slab as follows

$$
\begin{aligned}
& T(y, h, t)=T_{0} ;|y|<L, \\
& T(y,-h, t)=0 .
\end{aligned}
$$

From Dhaliwal and Singh [16], the following data (in SI unit) of Cobalt have been employed

$$
\begin{aligned}
& A_{22}=3.071 \times 10^{11} ; \quad A_{23}=1.027 \times 10^{11} ; \quad A_{33}=3.581 \times 10^{11} ; \quad A_{44}=1.510 \times 10^{11} ; \\
& h=1 ; \quad a=0.05 ; \quad \omega=1 ; \quad \rho=8836 ; \quad \beta_{1}=7.04 \times 10^{6} ; \quad \beta_{2}=7.04 \times 10^{6} ; \quad \beta_{3}=6.9 \times 10^{6} ; \\
& T_{0}=298 ; \quad c_{e}=427 ; \quad k_{z}=69 ; \quad k_{y}=69 .
\end{aligned}
$$

\section{Concluding remarks}

1. Figure 2: The absolute value of $V$ decreases as $\mathrm{z}$ increases. The nature of $V$ is the same for three different cases CTE, ETE and TRDTE when $-1 \leq z \leq 0.2 . V$ is maximum when $z=0.8$ for the cases CTE and ETE.

2. Figure 3: The absolute value of $W$ is maximum for the case TRDTE for different values of $z$. The nature of displacement is almost the same with respect to wave propagation for the cases CTE and ETE.

3. Figure 4: The temperature is maximum of the heated region and gradually decreases within the region $-1 \leq z \leq 0.6$. The nature of temperature for the cases CTE, ETE and TRDTE is the same with respect to wave propagation.

4. Figure 5: The nature of the graph for three different cases CTE, ETE and TRDTE is symmetric about $y=0$. The absolute value of $\tau_{z z}$ gradually increases as $\mathrm{z}$ increases. It is extensive in nature.

5. Figure 6: The absolute value of $\tau_{y z}$ gradually decreases as $z$ increases. The nature of the graph is symmetric about $y=0$. It is contractive in nature.

6. Figure 7: For all values of time $t$, the absolute value of $\tau_{z z}$ gradually decreases as $\Omega$ increases for a fixed value of $z=0.5$. For fixed rotation numerical values of $\tau_{z z}$ remains same for all time $t$.

7. Figure 8: For fixed rotation, $\tau_{y z}$ gradually decreases as time $t$ increases for a fixed value of $z=0.5$. For a fixed time $t$, the nature of $\tau_{y z}$ is all most the same for different values of $\Omega$. 


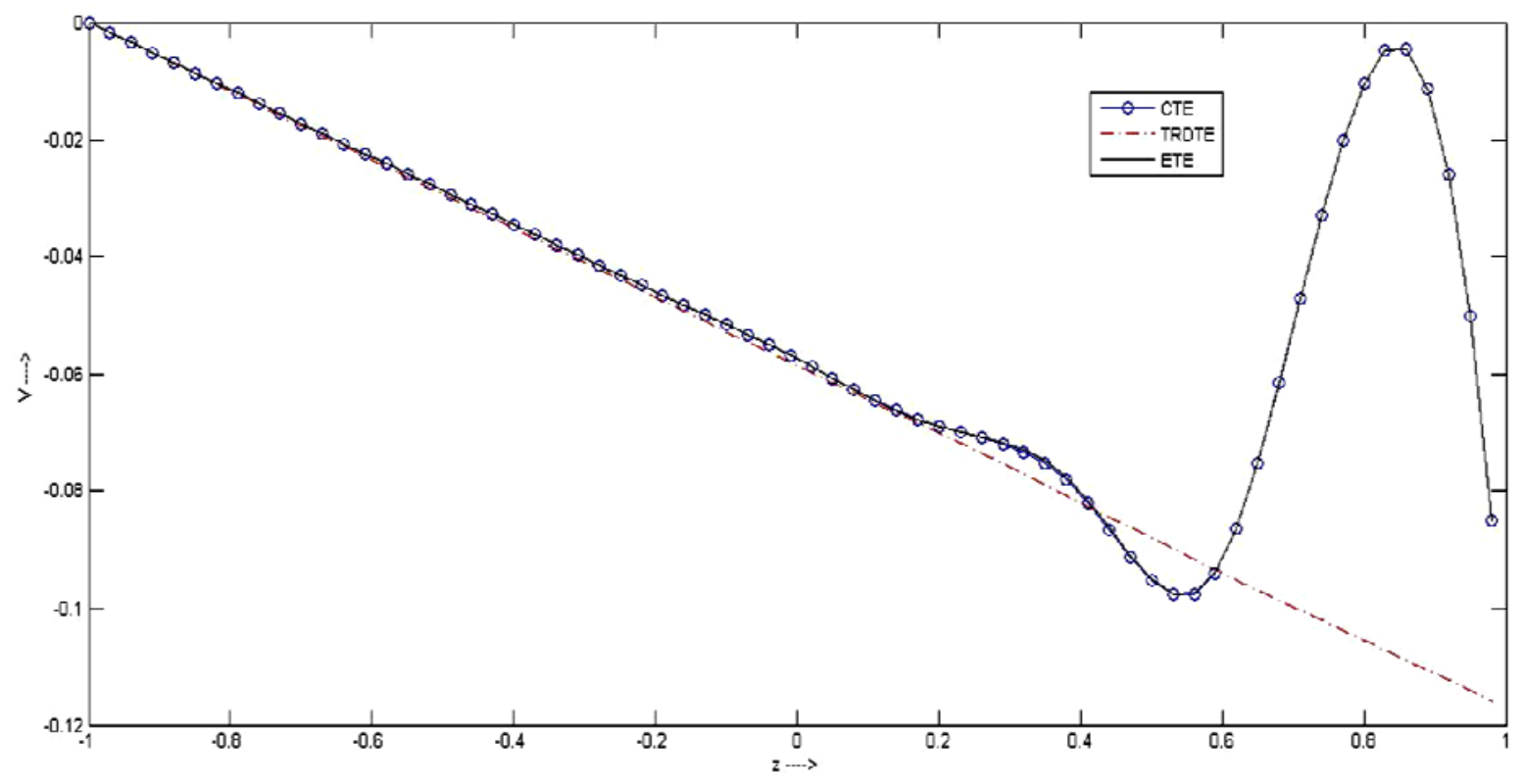

Fig.2. Variation of $V$ with respect to $z$, for a fixed value of $t=0.8$.

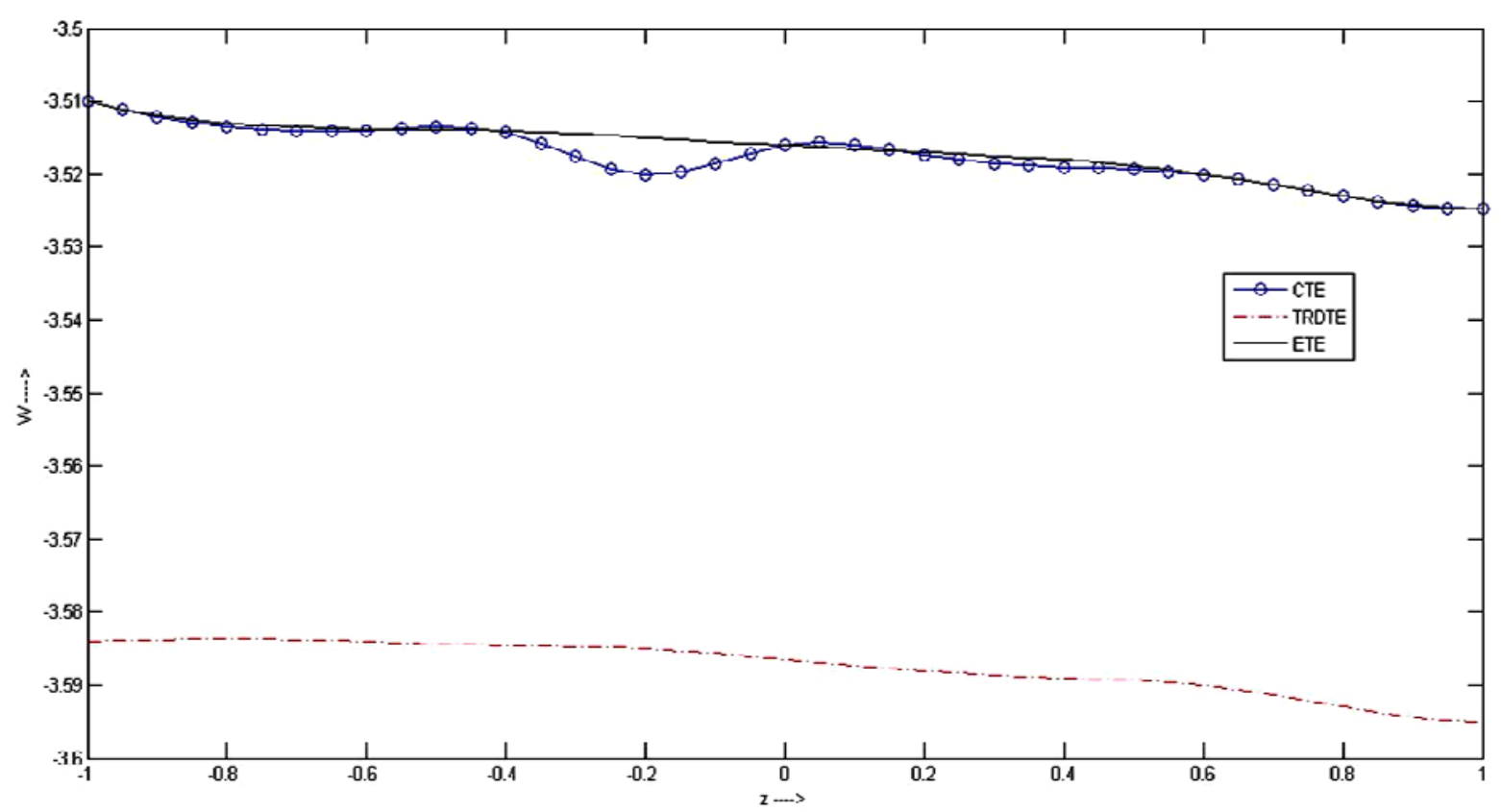

Fig.3. Variation of $W$ with respect to $z$, for a fixed value of $t=0.8$. 


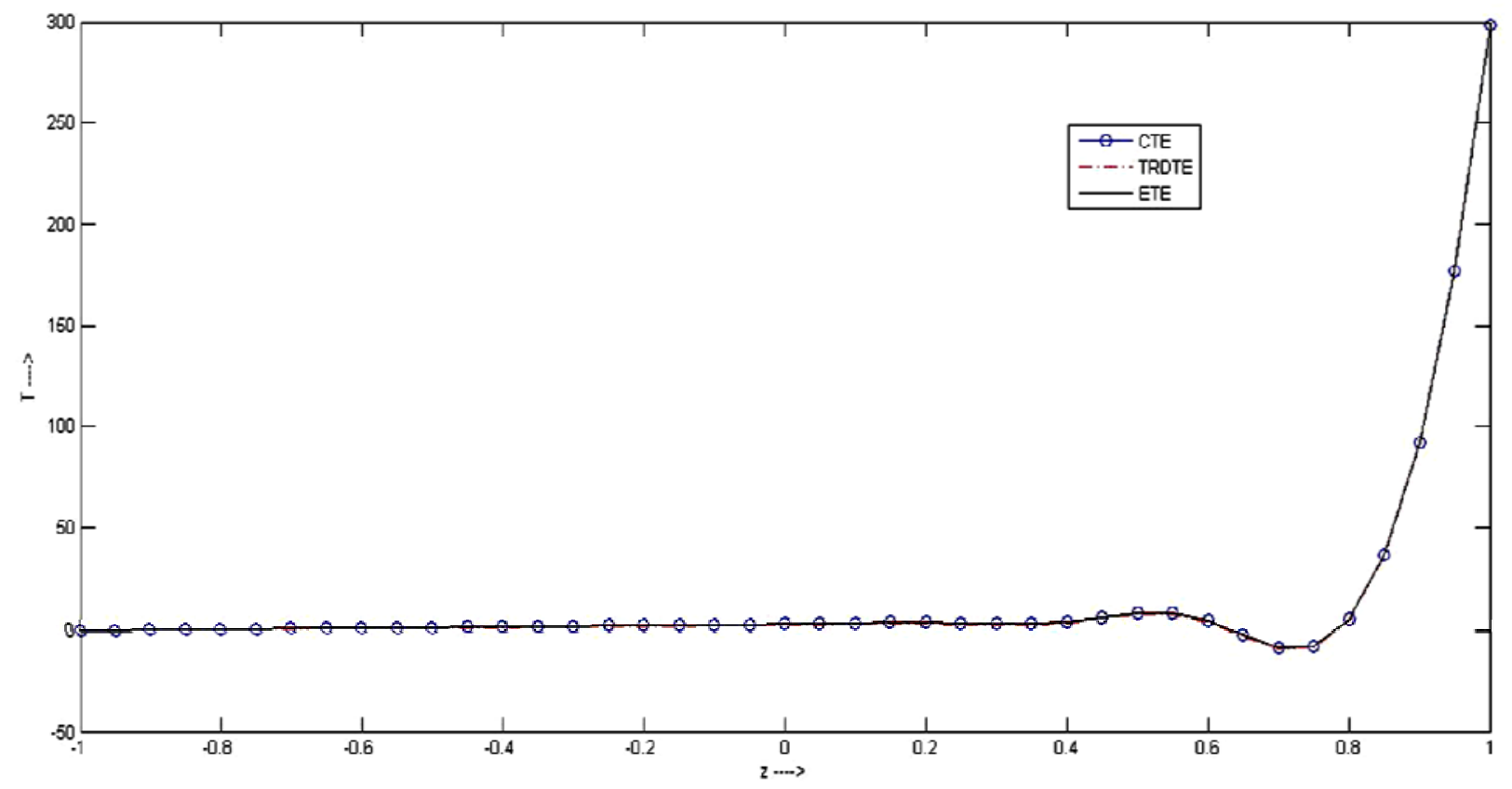

Fig.4. Variation of $T$ with respect to $z$, for a fixed value of $t=0.8$.

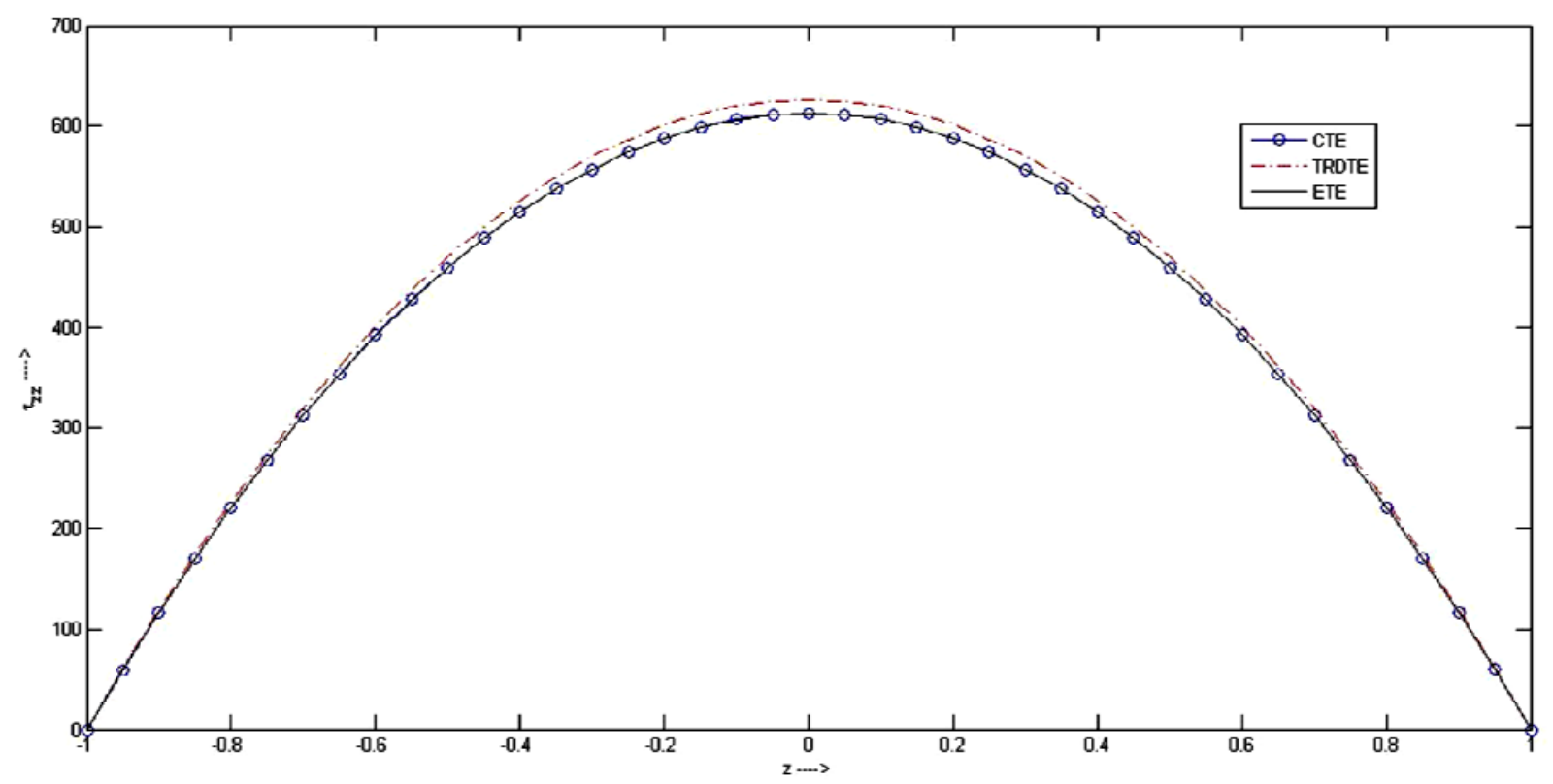

Fig.5. Variation of $\tau_{z z}$ with respect to $z$, for a fixed value of $t=0.8$. 


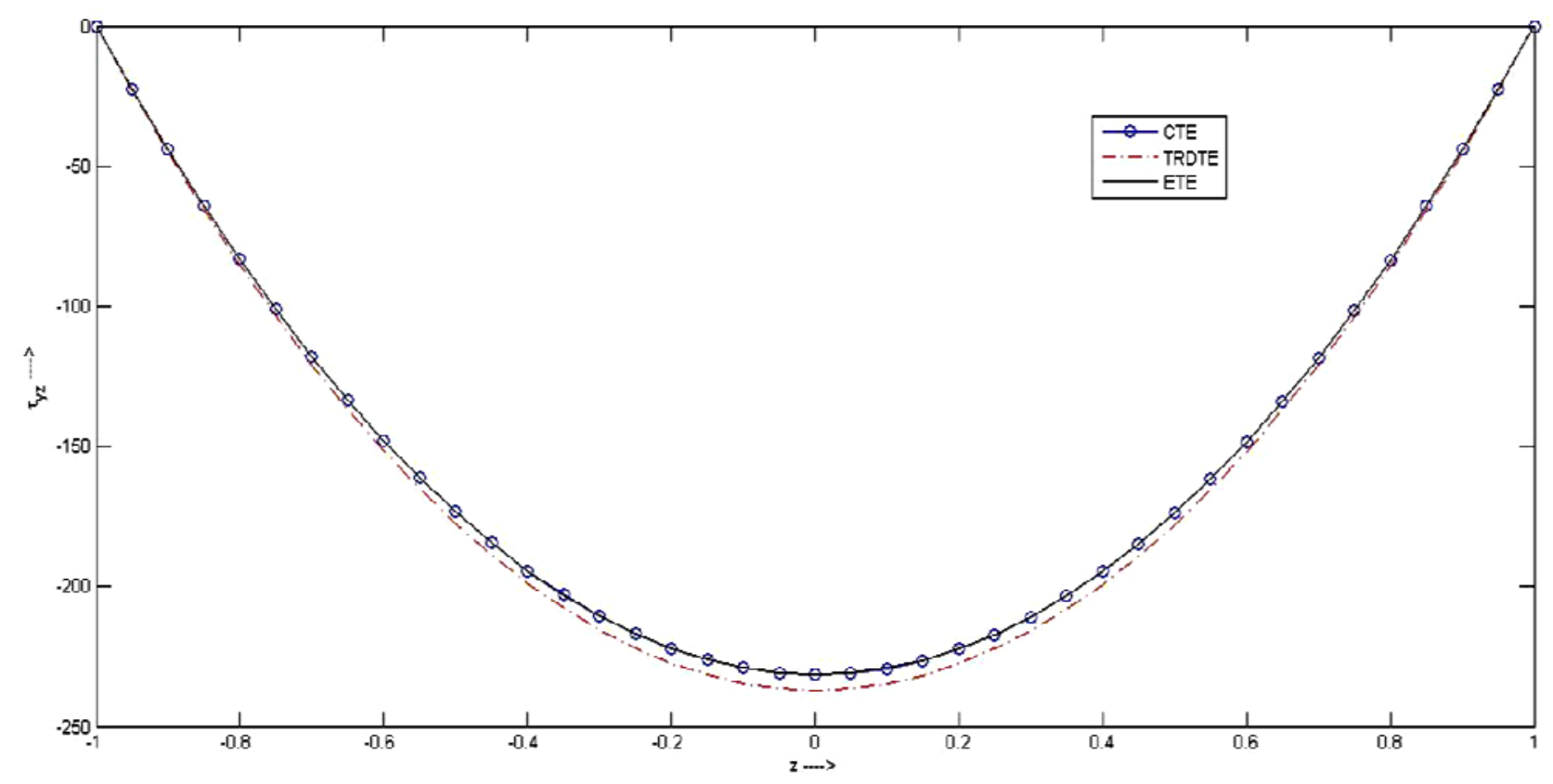

Fig.6. Variation of $\tau_{y z}$ with respect to $z$, for a fixed value of $t=0.8$.

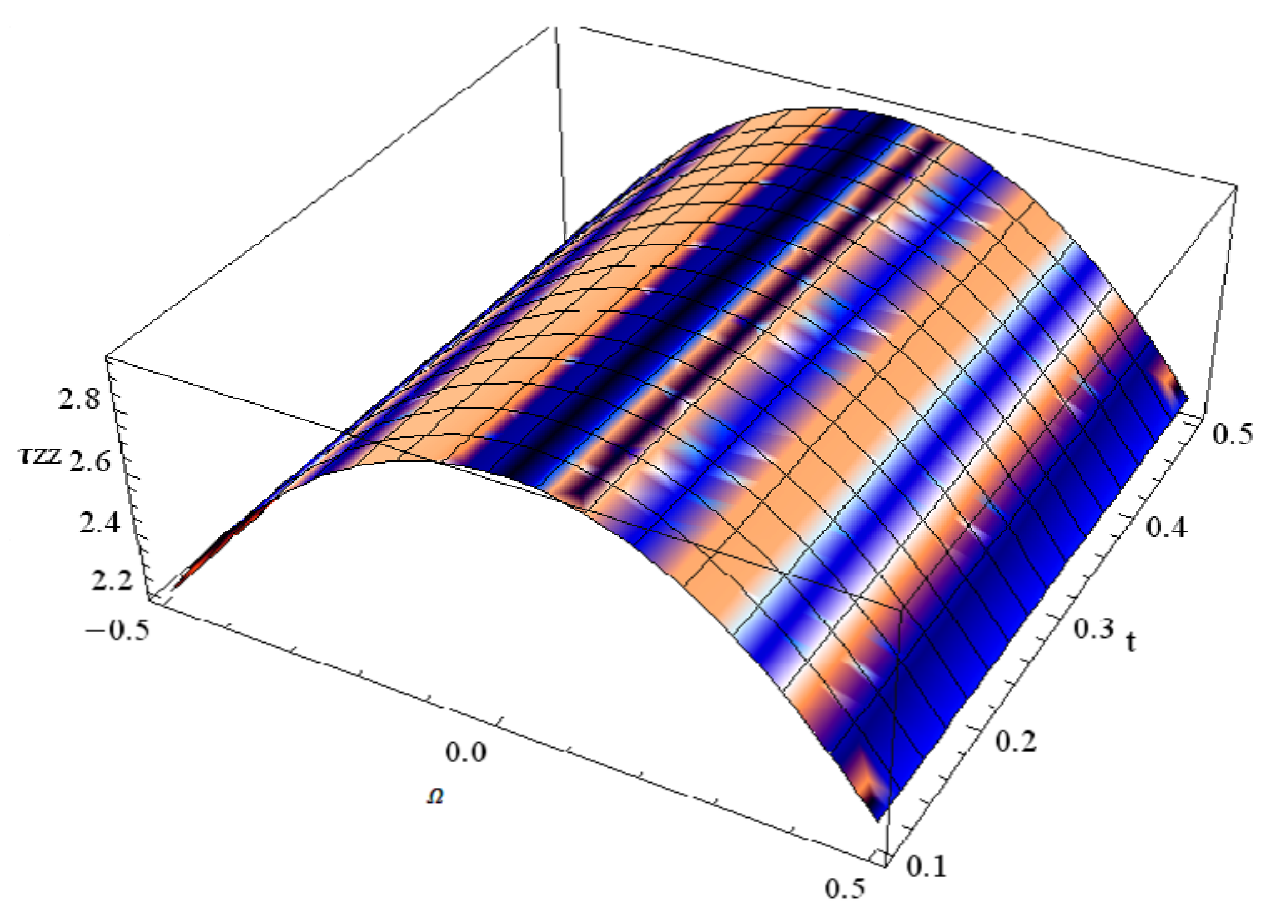

Fig.7. Variation of $\tau_{z z}$ with respect to $\Omega$ and $t$, for a fixed value of $z=0.5$. 


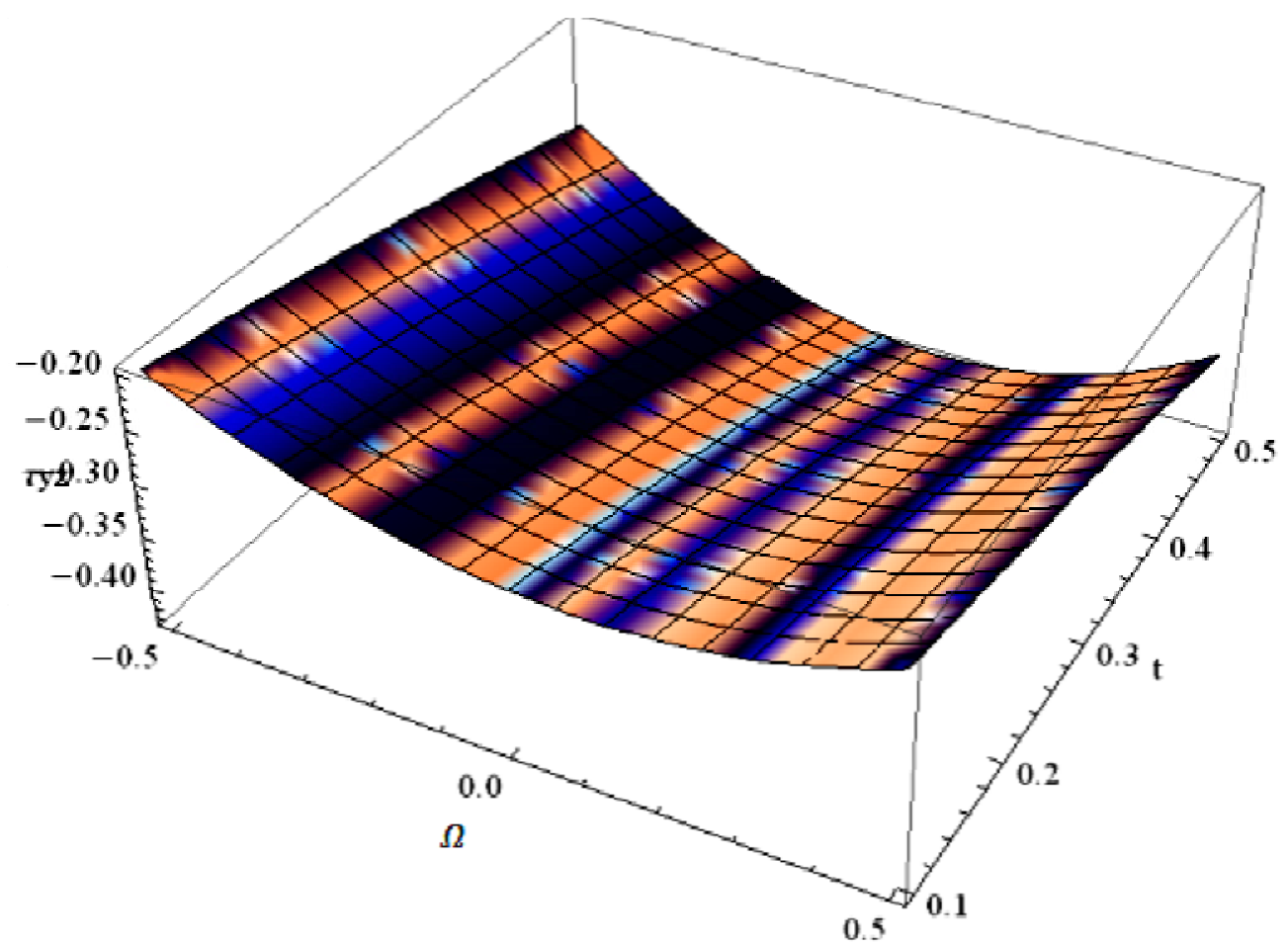

Fig.8. Variation of $\tau_{y z}$ with respect to $\Omega$ and $t$, for a fixed value of $z=0.5$.

\section{Acknowledgment} University.

The authors are thankful to DST - PURSE program of the Department of Mathematics in Jadavpur

\section{Nomenclature}

$$
\begin{aligned}
A_{i j k l} & - \text { elastic tensor } \\
a & \text { - wave number } \\
c_{e} & - \text { specific heat a constant strain } \\
e_{i j} & - \text { strain tensors } \\
k_{i j} & - \text { thermal conductivity tensor } \\
\boldsymbol{u} & - \text { displacement vector } \\
\alpha_{k l} & - \text { thermal expansion tensor } \\
\delta_{i j} & - \text { Kronecker delta } \\
\rho & - \text { density } \\
\tau & - \text { thermal relaxation time. } \\
\tau_{i j} & - \text { stress tensors } \\
\Omega & - \text { rotation } \\
\omega & - \text { angular frequency }
\end{aligned}
$$




\section{References}

[1] Bachher M., Sarkar N. and Lahiri A. (2015): Fractional order thermoelastic interactions in an infinite porous material due to distributed time-dependent heat sources. - Meccanica, vol.50, pp.2167-2178.

[2] Biot M.A.(1956): Thermoelasticity and irreversible thermodynamics. - J. Appl. Phys., vol.27, pp.240-253.

[3] Das B. and Lahiri A. (2015): Generalized magnetothermoelasticity for isotropic media. - Journal of Thermal Stresses, vol.38, No.2, pp.210-228.

[4] Das N.C., Lahiri A. and Giri R.R. (1997): Eigenvalue approach to generalized thermoelasticity. - Indian J. Pure Appl. Math., vol.28, No.12, pp.1573-1594.

[5] Dhaliwal R.S. and Sherief H.H. (1980): Generalized thermoelasticity for anisotropic media. - Quart. Appl. Math., vol.33, pp.1-8.

[6] Green A.E. and Lindsay K.A. (1972): Thermoelasticity. - Journal of Elasticity, vol.2, pp.1-7.

[7] Ignaczak J. (1979): Uniqueness in generalized thermoelasicity. - Journal of Thermal Stresses, vol.2, pp.171-175.

[8] Ignaczak J. (1982): Uniqueness in generalized thermoelasicity with one relaxation time. - Journal of Thermal Stresses, vol.5, pp.275-263.

[9] Kar A. and Kanoria M. (2006): Thermoelastic interaction with energy dissipation in an infinitely extended thin plate containing a circular hole. - Far East J. Appl. Math., vol.24, pp.201-217.

[10] Lord H.W. and Shulman Y. (1967): A generalized dynamic theory of thermoelasticity. - J. Mech. Phys. Solids, vol.15, pp.299-309.

[11] Sherief H.H. (1987): On uniqueness and stablity in generalized thermoelasticity. - Quart Appl. Math. (USA), vol.44, pp.773-778.

[12] Sherief H.H. (1986): Fundamental solution of the generalized thermoelastic problem for the short times. Journal of Thermal Stresses, vol.9, pp.151-164.

[13] Sherief H.H. and Anwar M.N. (1986): Problems in generalized thermoelasticity. - Journal of Thermal Stresses, vol.9, pp.165-181.

[14] Sherief H.H. and Dhaliwal R.S. (1980): A uniqueness theorem and a varational principle for generalized thermoelasticity. - Journal of Thermal Stresses, vol.3, pp.223-230.

[15] Tauchert T.R. and Akoz A.Y. (1974): Thermal stresses in an orthotropic elastic slab due to prescribed surface temperature. - J. Appl. Mech., vol.41 (Series E), pp.222-228.

[16] Dhaliwal R.S. and Singh A. (1980): Dynamic Coupled Thermoelasticity. - Hindustan Publ., Delhi.

Received: February 29, 2016

Revised: October 21, 2016 